\title{
Reinforcing loose foundation stones in trait-based plant ecology
}

\author{
Bill Shipley ${ }^{1}$ Francesco De Bello ${ }^{2,3} \cdot$ J. Hans C. Cornelissen $^{4} \cdot$ Etienne Laliberté5 $^{\text {. }}$ \\ Daniel C. Laughlin ${ }^{6}$ Peter B. Reich ${ }^{7,8}$
}

Received: 2 March 2015 / Accepted: 4 January 2016 / Published online: 21 January 2016

(C) Springer-Verlag Berlin Heidelberg 2016

\begin{abstract}
The promise of "trait-based" plant ecology is one of generalized prediction across organizational and spatial scales, independent of taxonomy. This promise is a major reason for the increased popularity of this approach. Here, we argue that some important foundational assumptions of trait-based ecology have not received sufficient empirical evaluation. We identify three such assumptions and, where possible, suggest methods of improvement: (i) traits are functional to the degree that they determine individual fitness, (ii) intraspecific variation in functional traits can be largely ignored, and (iii) functional traits show general predictive relationships to measurable environmental gradients.
\end{abstract}

Keywords Comparative ecology · Functional ecology · Intraspecific variation $\cdot$ Environmental gradients

Communicated by Fernando Valladares.

Bill Shipley

Bill.Shipley@USherbrooke.ca

Daniel C. Laughlin

d.laughlin@waikato.ac.nz

1 Département de Biologie, Université de Sherbrooke, Sherbrooke, QC J1K 2R1, Canada

2 FdB Institute of Botany, Academy of Sciences of the Czech Republic, Dukelská 135, 37982 Třeboň, Czech Republic

3 Department of Botany, University of South Bohemia, Na Zlaté Stoce 1, 37005 České Budějovice, Czech Republic

4 Systems Ecology, Department of Ecological Science, FALW, VU University, De Boelelaan 1085, 1081 HV Amsterdam, The Netherlands

\section{Introduction}

Most ecology textbooks differentiate between basic subdisciplines such as physiological, population, community, and ecosystem ecology. Researchers working in these different subdisciplines measure different variables that are designed to answer research questions pertaining to different and distinct phenomena, making it difficult to integrate our understanding of ecology across organizational scales. Despite such conceptual challenges, there are obvious links between these subdisciplines. Differences in morphology, chemistry, physiology, and phenology affect the ability of individuals to grow, survive, reproduce, and disperse in a given environment (i.e., individual fitness and demography). Differences in these demographic properties affect population dynamics. Differential population dynamics among coexisting species determine community composition and structure. Finally, all of these affect ecosystem properties because they affect the capture, loss, and cycling of energy and resources. Such changes in ecosystem

5 Institut de Recherche en Biologie Végétale (IRBV), Université de Montréal, 4101 Sherbrooke Est, Montréal, QC H1X 2B1, Canada

6 Environmental Research Institute and School of Science, University of Waikato, Private Bag 3105, Hamilton 3240, New Zealand

7 Department of Forest Resources, University of Minnesota, St. Paul, MN 55108, USA

8 Hawkesbury Institute for the Environment, University of Western Sydney, Penrith, NSW 2751, Australia 
properties can then change the fitness consequences of traits.

Such integration might be possible via another subdiscipline whose definition is so ambiguous that it has been renamed at least three times. The older name was "comparative ecology" (Grime 1965, 1979). A new name was introduced in 1987 when the British Ecological Society launched the journal Functional Ecology and a number of leading ecologists attempted to define the newly named subdiscipline of "functional" ecology in its first issue (e.g., Calow 1987). As might be expected, no consensus was reached. One leading ecologist (Bradshaw 1987) even questioned whether the newly baptised functional ecology was simply a rebranding of the older comparative ecology. As an example of this ambiguity, Grime et al.'s (1988) book even has both words in the title. The term "functional ecology" now seems to be waning and it has become popular to talk of "trait-based ecology." This new type of ecology seems curiously similar to the older functional ecology.

One reason for its existential identity crisis is because this subdiscipline is not defined by the phenomena that it studies or the organizational scale at which it is studied but rather by the way that it studies them. The defining attributes of this subdiscipline are fourfold. The first is a description of organisms that emphasizes the values of their phenotypic traits over their taxonomic or phylogenetic affiliations (thus "trait-based" ecology). The second defining attribute is the explicit comparison of trait values between many species and environments in order to elucidate general trends that are not limited to narrow taxonomic groups or geographical locations (thus "comparative" ecology). The third defining attribute is the comparison of these trait values along environmental gradients (including biotic components of these) to understand both how different trait combinations affect the environment ("effect" traits) and how different environments select different trait combinations ("response" traits). The fourth attribute is an explicit scaling of traits, or composites of these, from plant modules to entire ecosystems by assuming that that structure and function at higher organizational scales is largely a result of the composite traits of the individuals present (Lavorel and Grigulis 2012; Lavorel et al. 2011; Reich 2012). This view that plants function in communities and ecosystems via their traits leads to the notion of "functional" ecology. Grime (2001, page xvi) states that the emphasis of function over evolutionary affiliation (i.e., taxonomy) “... may yet prove to have been a crucial step in defining the nature and method of ecological enquiry." This was (Harper 1982), and perhaps still is, a controversial view. For simplicity, we will call this subdiscipline "trait-based ecology" while acknowledging the close links to the older terms.

The promise of trait-based ecology is in synthesis, integration, and generalized prediction (Reich et al. 1997;
Shipley 2007). By concentrating on generalizable properties of organisms (traits) and environments one should be able to move between scales of biological organization and between geographical locations to predict community structure and assembly, ecosystem-level phenomena such as rates of productivity and decomposition, the cycling of nutrients and energy, and ecosystem services, despite the idiosyncratic details of each site, taxonomic assemblage, or geographical location. This promise of generality, synthesis, and predictive ability, together with the increasing availability of trait values in the literature, are perhaps the major reasons why there has been an explosion of research papers using this approach in recent years. As often happens in rapidly advancing areas of research, there is a tendency to attack those problems that seem the most soluble or novel while ignoring those problems that seem more difficult. As also often happens, there is a tendency to forget the intellectual history which has shaped our current understanding. The danger, when the more difficult problems are foundational claims or assumptions, is that the foundation will not support the construction and the motivating goals of generality, synthesis, and predictive ability will be missed. Because trait-based ecology has been most popular in plant ecology, our focus here is on plants. However, in principle, trait-based ecology is applicable to all organisms. With this in mind, the objective of this paper is to highlight three incompletely developed or poorly tested foundational assumptions that are slowing our progress towards the goals of integration and prediction and, where possible, to suggest ways forward. There are many other important weaknesses and unanswered questions in our science that we do not discuss because of lack of space. In particular, the linking of traits to ecosystem processes via effect traits involves additional foundational assumptions that will not be discussed here. The three foundational assumptions evaluated here are that (i) traits are functional to the degree that they determine individual fitness; (ii) that intraspecific variation in functional traits can be largely ignored; and (iii) that functional traits show general predictive relationships to measurable environmental gradients. Figure 1 illustrates these three assumptions.

\section{Traits are functional to the degree that they determine individual fitness}

The TRY global database of plant functional traits (Kattge et al. 2011) currently contains measurements of about 1100 traits and 100,000 plant species. Most of these traits are represented by relatively few studies, while a small number of traits, including specific leaf area, seed size, and a few others, are very commonly measured. Why is this? What is the justification for including or excluding a trait in any 
(A) Trait values determine individual fitness

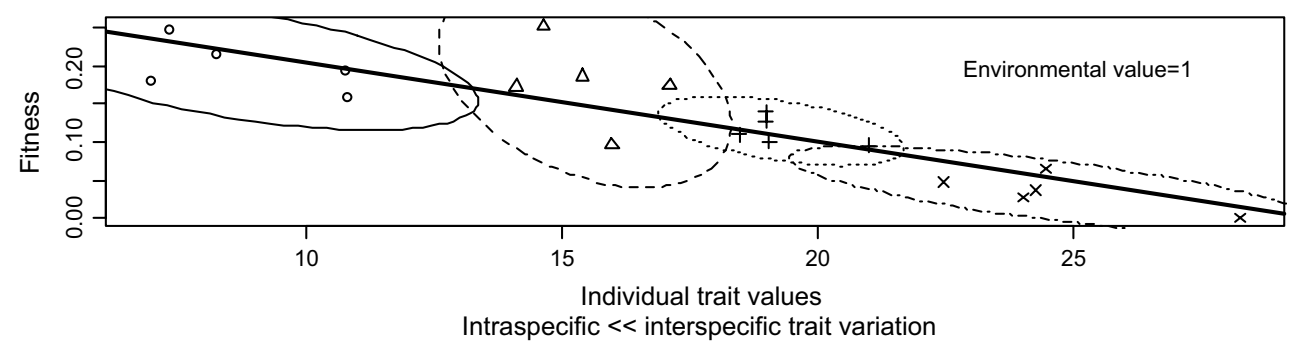

(B) Fitness trait relationships are environmentally dependent and result in species filtering

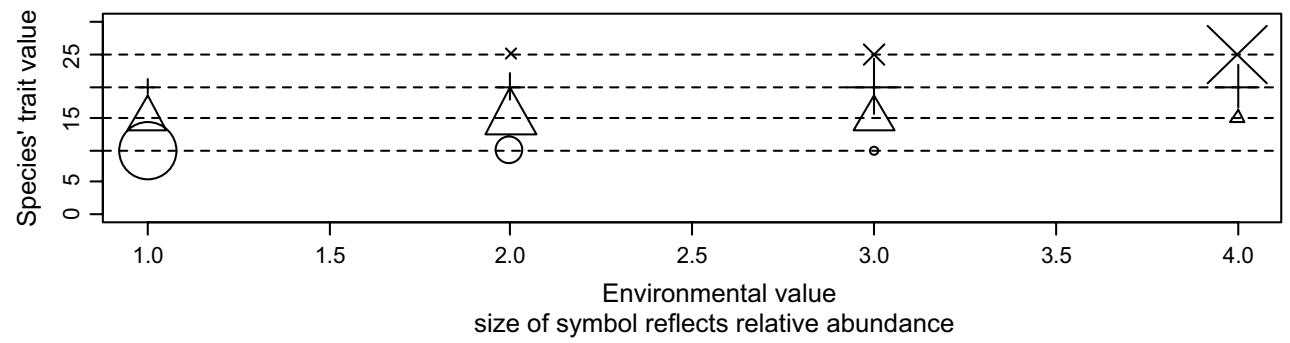

(C) Community-averaged trait values vary predictably with environment

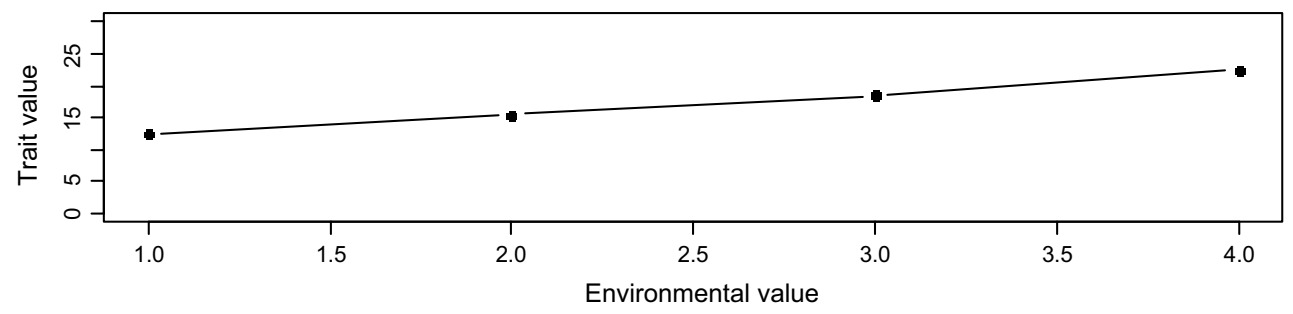

Fig. 1 a Fitness decreases with increasing values of a single trait. Plotted are the fitness values of 20 individuals belonging to four different species that are growing in a single site with an environmental value of 1. Ellipses show the range of values for each species; note that intraspecific trait variation is less than interspecific trait variation. b The relative abundance of each of these four species (shown by different symbols) in each of four different environments reflects the average fitness of its individuals. The species with the smallest trait value (circles) is dominant in the site with an environmental value of 1 because its members have the highest fitness. The opposite

research project? Since the main goal of trait-based ecology is to allow the prediction of ecological phenomena across species, sites, and environments, this goal should motivate our choice of traits. If there is a mismatch between this goal and our chosen traits then this weakens the foundation of trait-based ecology.

One obvious criterion for the inclusion of a trait is ease and speed of measurement. This is reasonable since a trait that is too expensive or time-consuming cannot be measured on many species in many sites, which is one of the requirements for generality. However, an easily measured trait is not necessarily a functionally important one. Presumably, researchers are attempting to trade off functional importance with ease of measurement, yet we have no relationship between trait values and fitness occurs in a site with an environmental value of 4 , where higher trait values confer higher fitness and so the species with the highest average trait values (multiplication symbols) is dominant. c Because small trait values confer the highest fitness in a site with an environmental value of 1 , most individuals belong to the species denoted by circles and so the community-averaged trait value is 12.5 . Because large trait values confer the highest fitness in a site with an environmental value of 4 , most individuals belong to the species denoted by multiplication symbols, so the average trait value is 23.5

good way of quantifying "functional importance" and, in any case, the degree of functional importance of most traits will be context specific. Virtually every phenotypic property of an organism will have a functional consequence in at least some environments, so it is not enough to rely on qualitative (yes/no) results from individual studies or intuitive arguments of cause and effect. We need better guides to trait selection for different types of ecological questions, particularly with regard to the type(s) of environmental gradient(s) studied.

One guide to trait selection is derived from the definition of Violle et al. (2007), who propose that different values of a "functional" trait must differentially affect the demographic probabilities of immigration, growth, 
survival, and reproduction (and thus fitness) of organisms possessing them. In Fig. 1a we see a general relationship in which fitness decreases with increasing values of a trait in a site with an environmental value of 1 . Therefore, this trait is "functional." This definition is more appropriate for response traits than for effect traits since there is no logical reason why a trait could not strongly affect certain properties of the external environment while having only minor consequences for individual fitness. A closer look at this definition points to useful suggestions for the selection of traits and to an important knowledge gap.

Absolute fitness in a given environment with respect to a heritable trait or combination of traits (i.e., a heritable phenotype) is the expected (population-averaged) lifetime reproductive output of an individual possessing it. One consequence of the above definition is that a trait cannot be simply classified as "functional" vs. "nonfunctional;" some traits are more functional than others in a given environment. The more strongly a trait determines fitness or components of it (growth, survival, reproduction, or dispersion), the more functional (and, presumably, the more useful) the trait. The strength of the correlation between trait values and fitness should guide our choice of traits. Unfortunately, with only a few exceptions (Adler et al. 2014; Chu et al. 2014; Kraft et al. 2010; Lasky et al. 2014; Ozinga et al. 2007; Poorter et al. 2008; Russo et al. 2010; Wright et al. 2010), we have very limited information about the strength of correlations between traits and fitness, as opposed to proxies or components of fitness like growth rates or various forms of physiological tolerance. Although almost all trait-based studies implicitly assume a relationship such as the one shown in Fig. 1a, we have surprisingly few such figures derived from empirical data. Worse, no studies have measured how the strength of correlations between traits and fitness vary across environments for commonly used functional traits. This is particularly worrisome because a central property of natural selection is that the fitness value of a particular trait combination varies strongly with the environment. This is also a central assumption of Keddy's (1992) notion of species filtering (Fig. 1b), which is simply a species-level consequence of individual natural selection based on traits plus the fact that at least some variation in functional traits is interspecific (Shipley 2010a). In other words, individuals whose traits confer higher fitness will leave more offspring and the species to which these better-adapted individuals belong will increase in relative abundance. Worse still, the limited available information directly linking traits and fitness refers primarily to fitness with respect to abiotic components of the environment rather than biotic components; an exception is Kraft et al. (2015). Although such field research linking traits and fitness is vital and important in itself, the difficulty involved in measuring demographic parameters for many different species in many different environments means that it is unlikely that such research can provide practical guidance in evaluating the trade-off between functional importance and ease of measurement. Despite its foundational importance, the practical difficulty of documenting trait-fitness relationships means that we cannot use this as a practical criterion for choosing traits. As a practical matter, we must rely either on proxies of fitness or on variables that are believed to be important components of it. Given this, it is critical that we empirically document the strengths between fitness and these other variables in order to judge the "functional" importance of traits.

One useful proxy of fitness that is much easier to measure in the field is relative abundance. Trait values associated with greater fitness in a given environment must, on average, correlate positively with the relative abundance of the species possessing them in that environment. A species with a higher relative abundance will usually have a relatively high number of individuals; this is true by definition if the unit of abundance is the number of individuals, and so this would be the preferred unit when inferring fitness. Except for demographic stochasticity (important in small populations or when fitness differences between species are small) or strong immigration from an external source population despite local maladaptation, a higher relative abundance can only arise because individuals of such species have higher probabilities of surviving, reproducing, and growing. Therefore, a more indirect way of judging the strength of trait-fitness correlations, and of the degree of "functionality" of different trait values, is to measure the correlation between the relative abundance of different species in a particular environment and their trait values. It is certainly easier to measure relative abundance than to directly measure fitness. Furthermore, there are now statistical methods for testing and quantifying relationships between trait values and relative abundances (Dray and Legendre 2008; Laughlin and Laughlin 2013; Shipley 2010a, b; Sonnier et al. 2012; Warton et al. 2014), including the ability to separate the effect of trait filtering on relative abundance from that caused by demographic stochasticity and immigration pressure (Shipley 2014). This might be a more practical, although indirect, way of judging the degree of "functionality" of traits and of choosing traits (Laughlin 2014). Certainly, as in every observational method, a correlation between trait values and relative abundance does not necessarily mean a causal link between them, so such studies must be augmented with methods such as structural equation modeling that allow for more sensitive tests of causal structure.

Another method (Grime 1965) of determining the degree of "functionality" of traits is through comparative experiments in which many species with contrasting trait values are grown in a common controlled environment (or a series 
of these). Fitness, or components of fitness such as growth rate, survival, or reproductive output, is measured for each species and correlated with the trait values. This method is easier than measuring fitness in the field and has the added advantage that the "environment" is clearly defined and measured. There are also important disadvantages. First, this method is still too expensive and time-consuming to include more than a small number of species and environments, which severely reduces the level of generality of the results. Second, while this method is well suited to studying relationships between traits and purely abiotic environmental conditions, it is much more difficult to design such experiments when fitness is influenced by biotic interactions, which is a common occurrence. Third, a proper evaluation of fitness may require more than a single generation of plants in order to account for plant-soil feedbacks or transient dynamics. Finally, given the difficulty of extrapolating results from the lab to the field, this method cannot really answer the most important question when judging the functional importance of traits: how strong is the link between trait values and fitness in the field environment?

Probably the most common justifiable shortcut is to identify certain ecological "abilities" that can reasonably be related to fitness in a given environment and then to choose traits that we suspect are important determinants of these abilities. When dealing with environments for which there is a single overriding selective force it is reasonable to assume that traits which are known to be important adaptations to this selective force will also be strongly correlated with fitness. However, most habitats have several selective forces whose relative importance is unknown and likely variable in space and time.

There is a large ecophysiological literature (for example, Lambers et al. 1998) relating traits to various ecological abilities (for example, tolerance to water shortages, cold or hot temperatures), although this literature is strongly biased towards abiotic variables rather than biotic interactions. Perhaps the best-documented example relates to differences in light availability, for which there is a good literature linking various traits to differences in the typical light environments of species (Poorter and Bongers 2006; Poorter et al. 2010; Sterck et al. 2006; van Gelder et al. 2006; Walters and Reich 1996). However, there are still important knowledge gaps in moving from such ecophysiological studies to field-based studies involving many different species and environments. First, since we often do not know the relative importance of different ecological abilities in determining fitness in different environments, or the multivariate causal network linking traits to ecological abilities, then we still risk choosing traits that are only weakly associated with fitness. Second, the traits measured by ecophysiologists are often too difficult and time-consuming to measure on large numbers of species. A common shortcut is to identify easily measured ("soft") traits that are correlated with more demanding ("hard") traits using the implicit causal logic: soft traits $\rightarrow$ hard traits $\rightarrow$ fitness. Unfortunately, unless we can identify combinations of soft traits that are strongly correlated to the hard traits which are, in turn, strongly correlated to fitness then we have only compounded our problem since we will have further weakened the correlation between our traits and fitness.

It is not at all clear that the frequency with which different traits are measured in the literature reflects the strength of their links to individual fitness, and thus their functional importance, in the environments under study. The link between functional traits and fitness in a given environment is a foundational assumption of trait-based ecology and justifies much of its explanatory content. Each of the methods described above have strengths and weaknesses and all can contribute to this difficult problem. If this foundation stone is not reinforced by a strong empirical base then the entire construction of trait-based ecology is at risk.

\section{Intraspecific variation in functional traits is largely ignored}

With the introduction of trait-screening protocols by Grime (1965) and collaborators, which consisted of growing relatively few individuals of large numbers of contrasting species in common environments, came the criticism that such trait-based approaches ignore or downplay intraspecific variation and trait plasticity (Harper 1982). Grime's approach to trait screening has been carried forward into existing protocols for trait measurement in the field (for example, Cornelissen et al. 2003b; Hendry and Grime 1993; Pérez-Harguindeguy et al. 2013) that focus on relatively few standardized individuals (mature, exposed to full light, not affected by herbivores or pathogens) per species within one or a few environmental conditions. As a consequence, most studies in trait-based ecology use only a mean trait value per species.

The original justification for this approach (Grime 1979; Grime and Hunt 1975) was that, in broad interspecific comparisons, interspecific trait variation would be much greater than intraspecific trait variation, and that existing intraspecific trait variation would not obscure the broad trends. This assumption simplified work on the original motivating questions related to broad interspecific patterns because, given practical constraints of time and space, a reduction in intraspecific replication allowed a greater number of species to be studied, therefore providing a greater degree of generality. Both experimental (Hunt 1984; Meziane and Shipley 1999a, b, 2001) and field (Garnier et al. 2001) studies provided some empirical justification for this assumption, at least for some traits and types of environmental 
variation. A closely related assumption is that, even if intraspecific trait variation affects the quantitative interspecific relationships between traits, at least the interspecific ranking between species is maintained; this assumption has been confirmed in the relatively few studies that have tested it (Albert et al. 2010; Cornelissen et al. 2003a; Garnier et al. 2001; Kazakou et al. 2014; Makkonen et al. 2012; Roche et al. 2004; Rose et al. 2013). Figure 1a illustrates this assumption. The ellipses show the intraspecific variation in the trait-fitness relationship for each of four species. Since these ellipses are mostly nonoverlapping, we could replace the individual values by the bivariate species averages without losing too much information.

However, it is clear that some traits are more plastic than others. For instance SLA, one of the most reported functional traits, can double in value within a few hours following a reduction in irradiance (Shipley 2000b). Furthermore, such trait plasticity can be, in itself, a determinant of fitness, and this has been largely ignored in the literature of trait-based ecology. Besides plastic trait variation, there can also be ecotypic intraspecific variation.

As the degree of interspecific trait variation within a study decreases, the relative importance of intraspecific trait variation must increase. So, when can intraspecific trait variation be safely ignored and when must it be included? For which traits is this true? Along which environmental gradients? Are there certain habitat types for which intraspecific trait variation is sufficiently important that its exclusion is problematic? Only recently have such questions begun to be empirically studied (Albert et al. 2012, 2011; Auger and Shipley 2013; De Bello et al. 2011; Jung et al. 2014; Lepš et al. 2011; Violle et al. 2012) and the answers are still tentative and incomplete. If it was true that most functional traits had substantial levels of intraspecific variation, were highly plastic, and had values that changed idiosyncratically along environmental gradients, then this would not necessarily challenge the theoretical basis of trait-based ecology; it would only mean that one would need to measure trait values for each individual rather than relying on average values of species or sites. However, if one must work at the level of individuals, then functional ecology would largely forfeit the claim to generalized predictive ability that motivates it.

\section{Functional traits show general predictive relationships to measurable environmental gradients}

Another foundational claim is that similar environmental conditions will select for a similar distribution of response traits, and a similar distribution of effect traits will cause similar ecosystem effects, irrespective of taxonomic differences between sites. In other words, we should find systematic relationships between trait values and environmental variables that are generalizable across geographic locations and taxonomic composition.

Stated mathematically, we require a relationship of the form $f(x, y, z)=g(a, b, c)$, where $f(x, y, z)$ is some function of a series of traits and $g(a, b, c)$ is some function of a series of environmental variables (Fig. 1c). Critically, since the foundational assumption is between environments and traits independent of idiosyncratic differences between sites and taxonomic composition, this relationship must be general. In other words, we must be able to go to a new site, measure the same environmental variables $(a$, $b, c$ ), enter their values into the same mathematical function, $g(a, b, c)$, and successfully predict the distribution of trait values possessed by the plants in this new site. Only when we can do this can we claim to predict trait values from the environment (or vice versa). For instance, given Fig. 1c, if we find a site whose environmental value is 2.5 then we would be able to predict that the individual plants in this site have, on average, a trait value of 17.5. Sadly, we have very few empirical examples of such generalizable predictive relationships and this is because trait-based ecology cannot decide how to measure the environment in such a way as to convert it into a "gradient". If different studies used different definitions, protocols and methods when measuring the same "trait" then it would be impossible to compare between them; yet this is the case with respect to the mathematical functions (i.e., "gradients") linking abiotic variables to plant attributes.

There is a vast literature associated with the use of multivariate methods of gradient analysis (Legendre and Legendre 1998) to describe quantitative relationships between taxonomic composition (a species $\times$ site matrix of abundances) and environmental variables (an environment $\times$ site matrix of abiotic variables). Such studies certainly provide quantitative relationships between compositional similarity and environments, but they are not generalizable to new sites because new sites will have different species compositions and so do not have the properties that we require. An equivalent analysis in trait-based ecology would be an analysis of a trait $\times$ site matrix of community-weighted trait values with respect to an environment $\times$ site matrix of abiotic variables. There is no requirement, or even expectation, that the function linking these two matrices should be linear. If we have chosen traits that properly reflect fitness differences between individuals of different species, and environmental variables that properly measure the selective forces at work in these sites, and if our foundational assumption is correct, then the resulting trait-based environmental gradients will be generalizable and predictable. However, with few exceptions, we do not possess such generalizable and quantitative 
relationships relating trait values to environmental variables, so this foundational claim is also weakly supported. Certainly, several studies have documented significant relationships between traits and environmental conditions, such as climate and soil properties (for example, Hayes et al. 2014; McDonald et al. 2003; Ordoñez et al. 2009; Vitousek et al. 1995), which provide empirical support for the belief that traits and certain trait combinations are being selected along these gradients. However, the extent to which these patterns can be generalized beyond the local regions in which the studies were conducted is still unknown, and such generalization is a key foundational assumption. Why, given the foundational importance of such patterns, do we have so few examples of generalizable and quantitative trait-based environmental gradients? After all, there are many well-established methods and instruments for measuring many properties of the abiotic environment (for example, Carter 1993; Pearcy et al. 1991), and even online information on worldwide climatic conditions.

One reason might be because we have not properly conceptualized and identified the actual selective forces controlling trait variation. For instance, if a trait is being selected on the basis of variation in the average (or variance) of the supply rate of whatever soil nutrient is limiting growth, then measures of supply rates of a single soil nutrient (which would not always be limiting) or measures of soil concentrations of such nutrients (which might not reflect supply rates) or punctual measures (which might not reflect long-term values) will not be generalizable. An environmental variable whose predictive value is variable across sites would be a clue that we have not properly conceptualized and measured the true selective force in the environment. For instance, both Grime's (1979) and Tilman's (1988) theories of plant strategies claim that there are two primary environmental drivers of trait variation: levels of disturbance and stress, or closely related concepts like productivity or "fertility." If this claim is true then a generalizable trait-environment relationship would require that we are able to combine various proximate variables into a more general measure of "disturbance" or "stress". How can we compare our distributions of trait values at equal levels of "disturbance" and "stress" unless we can agree on which environmental variables we must measure, how we must measure them, and how we must combine them together to obtain comparable values of "disturbance" and "stress?" Even seemingly simpler concepts like "soil nutrient availability" or "soil water availability" lack clear definitions and methods of measurement that would allow us to generalize across many different sites worldwide. Part of the solution might be to include the older idea of common phytometers in the measurement definition (Beadle 1954; Scott and Groves 1989). This conceptual problem of measurement is similar to those encountered in the social sciences, in which variables that are believed to be causally important are not directly measureable (i.e., they are latent) while variables that are directly measurable are only imperfect indicators of the causally important ones. Perhaps the statistical methods developed in these sciences, involving measurement models and structural equation modeling (Grace 2006; Shipley 2000a), could move us forward? One example of this approach to measuring disturbance and soil fertility is Sonnier et al. (2010).

Another complication is that different selective forces can act on the same trait. If the strength of selection of these different environmental variables varies across sites, and if we have not measured all of the important variables, then the predictive ability of our measured variables will also be variable. This is an unavoidable statistical problem in any multivariate system, and further underlines the importance of identifying and properly measuring these selective forces.

A final reason why we have few generalizable traitenvironment relationships is perhaps because we have not invested enough effort in looking for them. There are now unprecedented efforts aimed at sharing trait information worldwide in order to improve the generality of our knowledge of trait interrelationships, and such collaborations have been fruitful (for example, Wright et al. 2004). These efforts have been successful because of a concerted effort to agree on common protocols for trait measurement and to pool results (Kattge et al. 2011). We desperately need accepted protocols for measuring major environmental gradients linking traits to basic selective forces and measurements of these that are deposited along with trait information. Once this is done then we can apply model calibration and validation methods across multiple regions and continents to identify the most general trait-environment relationships and to help us to better conceptualize and measure the important underlying selective forces driving these general relationships. Calibrating trait-environment relationships on one continent and using trait-based models to predict abundances of species on a different continent would be the strongest test of the generality of trait-environment relationships. If such tests fail, then re-evaluations of the fundamental assumptions of trait-based ecology are required.

The dominant school of plant ecology during the midtwentieth century was organized around the methods of plant population biology and demography (Harper 1977), an explicitly taxonomic rather than trait-based approach. John Harper, a leader of this school, stated that the results obtained by this approach, when compiled by researchers working on different species and sites, could allow: “...the development of ecology as a generalizing and predictive science" (Harper 1982). Despite the many important contributions of this school, Harper's hope for a generalizing and 
predictive science of plant ecology via population biology was not realized and, as a result, it has lost influence. If the foundations of the trait-based approach are not reinforced then it too will fail to deliver on its promise of generality and integration, and could suffer a similar fate.

Given the incredible diversity of plant species and habitats, a proper evaluation of the foundational assumptions of trait-based ecology cannot be obtained from only a few studies. On the contrary, this requires many replicated studies of these questions. Despite the fierce competition in our scientific journals for novelty, the importance of research aimed at these older, but foundational, assumptions of traitbased ecology are essential and should be recognized as such.

Acknowledgments This paper has benefited from the comments of Éric Garnier, Sandra Díaz, Phillip Grime, Sandra Lavorel, Hendrik Poorter, Ian Wright, and two anonomous reviewers. Financing was provided by an NSERC Discovery Grant to BS.

Author contribution statement BS wrote the first draft of this paper and coordinated the subsequent changes. All other authors contributed equally to the subsequent drafts.

\section{References}

Adler PB et al (2014) Functional traits explain variation in plant life history strategies. Proc Natl Acad Sci USA 111(2):740-745. doi:10.1073/pnas.1315179111

Albert CH, Thuiller W, Yoccoz NG, Douzet R, Aubert S, Lavorel S (2010) A multi-trait approach reveals the structure and the relative importance of intra- vs. interspecific variability in plant traits. Funct Ecol 24:1192-1201

Albert CH, Grassein F, Schurr FM, Vieilledent G, Violle C (2011) When and how should intraspecific variability be considered in trait-based plant ecology? Perspect Plant Ecol Evol Syst $13: 217-225$

Albert CH, de Bello F, Boulangeat I, Pellet G, Lavorel S, Thuiller W (2012) On the importance of intraspecific variability for the quantification of functional diversity. Oikos 121:116-126

Auger S, Shipley B (2013) Inter-specific and intra-specific trait variation along short environmental gradients in an old-growth temperate forest. J Veg Sci 24:419-428

Beadle NCW (1954) Soil phosphate and the delimitation of plant communities in eastern Australia. Ecology 35:370-375

Bradshaw AD (1987) Functional ecology = comparative ecology? Funct Ecol 1:71-72

Calow P (1987) Towards a definition of functional ecology. Funct Ecol 1:57-61

Carter MR (ed) (1993) Soil sampling and methods of analysis. CRC, Boca Raton

Chu $\mathrm{C}$ et al (2014) Life form influences survivorship patterns for 109 herbaceous perennials from six semi-arid ecosystems. J Veg Sci 25:947-954

Cornelissen JHC et al (2003a) Functional traits of woody plants: correspondence of species rankings between field adults and laboratory-grown seedlings? J Veg Sci 14:311-322

Cornelissen JHC et al (2003b) A handbook of protocols for standardised and easy measurement of plant functional traits worldwide. Aust J Bot 51:335-380
De Bello F et al (2011) Quantifying the relevance of intraspecific trait variability for functional diversity. Methods Ecol Evol 2:163-174

Dray S, Legendre P (2008) Testing the species traits-environment relationships: the fourth-corner problem revisited. Ecology 89:3400-3412

Garnier E et al (2001) Consistency of species ranking based on functional leaf traits. New Phytol 152:69-83

Grace JB (2006) Structural equation modeling and natural systems. Cambridge University Press, Cambridge

Grime JP (1965) Comparative experiments as a key to the ecology of flowering plants. Ecology 45:513-515

Grime JP (1979) Plant strategies and vegetation processes. Wiley, New York

Grime JP (2001) Plant strategies, vegetation processes, and ecosystem properties, 2nd edn. Wiley, New York

Grime JP, Hunt R (1975) Relative growth rate: its range and adaptive significance in a local flora. J Ecol 63:393-422

Grime JP, Hodgson JG, Hunt R (1988) Comparative plant ecology: a functional approach to common British species. Unwin Hyman, London

Harper JL (1977) Population biology of plants. Academic, London

Harper JL (1982) After description. In: Newman EI (ed) The plant community as a working mechanism. Blackwell Scientific, Oxford, pp 11-25

Hayes P, Turner BL, Lambers H, Laliberté E (2014) Foliar nutrient concentrations and resorption efficiency in plants of contrasting nutrient-acquisition strategies along a 2-million-year dune chronosequence. J Ecol 102:396-410

Hendry GAF, Grime JP (1993) Methods in comparative plant ecology. Chapman \& Hall, London

Hunt R (1984) Relative growth rates of cohorts of ramets cloned from a single genet. J Ecol 72:299-305

Jung V, Albert CH, Violle C, Kunstler G, Loucougaray G, Spiegelberger $T$ (2014) Intraspecific trait variability mediates the response of subalpine grassland communities to extreme drought events. J Ecol 102:45-53

Kattge J et al (2011) TRY - a global database of plant traits. Glob Change Biol 17:2905-2935

Kazakou E et al (2014) Are trait-based species rankings consistent across data sets and spatial scales? J Veg Sci 25:235-247

Keddy PA (1992) Assembly and response rules: two goals for predictive community ecology. J Veg Sci 3:157-164

Kraft NJB, Metz MR, Condit RS, Chave J (2010) The relationship between wood density and mortality in a global tropical forest data set. New Phytol 188:1124-1136

Kraft NJ, Godoy O, Levine JM (2015) Plant functional traits and the multidimensional nature of species coexistence. Proc Natl Acad Sci USA 112:797-802

Lambers H, Chapin FS, Pons TL (1998) Plant physiological ecology. Springer, New York

Lasky JR, Uriarte M, Boukili VK, Chazdon RL (2014) Trait-mediated assembly processes predict successional changes in community diversity of tropical forests. Proc Natl Acad Sci USA 111:5616-5621

Laughlin DC (2014) The intrinsic dimensionality of plant traits and its relevance to community assembly. J Ecol 102:186-193

Laughlin DC, Laughlin DE (2013) Advances in modeling trait-based plant community assembly. Trends Plant Sci 18:584-593

Lavorel S, Grigulis K (2012) How fundamental plant functional trait relationships scale-up to trade-offs and synergies in ecosystem services. J Ecol 100:128-140

Lavorel S et al (2011) Using plant functional traits to understand the landscape distribution of multiple ecosystem services. J Ecol 99:135-147 
Legendre P, Legendre L (1998) Numerical ecology, 2nd edn. Elsevier, Amsterdam

Lepš J, de Bello F, Šmilauer P, Doležal J (2011) Community trait response to environment: disentangling species turnover vs intraspecific trait variability effects. Ecography $34: 856-863$

Makkonen M et al (2012) Highly consistent effects of plant litter identity and functional traits on decomposition across a latitudinal gradient. Ecol Lett 15:1033-1041

McDonald PG, Fonseca CR, Overton JM, Westoby M (2003) Leafsize divergence along rainfall and soil-nutrient gradients: is the method of size reduction common among clades? Funct Ecol 17:50-57

Meziane D, Shipley B (1999a) Interacting components of interspecific relative growth rate: constancy and change under differing conditions of light and nutrient supply. Funct Ecol 13:611-622

Meziane D, Shipley B (1999b) Interacting determinants of specific leaf area in 22 herbaceous species: effects of irradiance and nutrient availability. Plant Cell Environ 22:447-459

Meziane D, Shipley B (2001) Direct and indirect relationships between specific leaf area, leaf nitrogen and leaf gas exchange. Effects of irradiance and nutrient supply. Ann Bot 88:915-927

Ordoñez JC, Van Bodegom PM, Witte JPM, Wright IJ, Reich PB, Aerts R (2009) A global study of relationships between leaf traits, climate and soil measures of nutrient fertility. Glob Ecol Biogeogr 18:137-149

Ozinga WA et al (2007) Local above-ground persistence of vascular plants: life-history trade-offs and environmental constraints. J Veg Sci 18:489-497

Pearcy RW, Ehleringer J, Mooney HA, Rundel PW (eds) (1991) Plant physiological ecology. Chapman \& Hall, London

Pérez-Harguindeguy $\mathrm{N}$ et al (2013) New handbook for standardised measurement of plant functional traits worldwide. Aust $\mathrm{J}$ Bot $61: 167-234$

Poorter L, Bongers F (2006) Leaf traits are good predictors of plant performance across 53 rain forest species. Ecology 87:1733-1743

Poorter L et al (2008) Are functional traits good predictors of demographic rates? Evidence from five neotropical forests. Ecology 89:1908-1920

Poorter L et al (2010) The importance of wood traits and hydraulic conductance for the performance and life history strategies of 42 rainforest tree species. New Phytol 185:481-492

Reich PB (2012) Key canopy traits drive forest productivity. Proc Biol Sci R Soc 279:2128-2134

Reich PB, Walters MB, Ellsworth DS (1997) From tropics to tundra: global convergence in plant functioning. Proc Natl Acad Sci USA 94:13730-13734

Roche P, Díaz-Burlinson N, Gachet S (2004) Congruency analysis of species ranking based on leaf traits: which traits are the more reliable? Plant Ecol 174:37-48

Rose L, Rubarth MC, Hertel D, Leuschner C (2013) Management alters interspecific leaf trait relationships and trait-based species rankings in permanent meadows. J Veg Sci 24:239-250

Russo SE, Jenkins KL, Wiser SK, Uriarte M, Duncan RP, Coomes DA (2010) Interspecific relationships among growth, mortality and xylem traits of woody species from New Zealand. Funct Ecol 24:253-262

Scott D, Groves RH (1989) Empirical measurement of environmental gradient in ecological surveys. N Z J Ecol 12:89-94

Shipley B (2000a) Cause and correlation in biology: a user's guide to path analysis, structural equations, and causal inference. Cambridge University Press, Cambridge

Shipley B (2000b) Plasticity in relative growth rate and its components following a change in irradiance. Plant Cell Environ 23:1207-1216

Shipley B (2007) Comparative plant ecology as a tool for integrating across scales. Ann Bot 99:965-966

Shipley B (2010a) Community assembly, natural selection and maximum entropy models. Oikos 119:604-609

Shipley B (2010b) Inferential permutation tests for maximum entropy models in ecology. Ecology 2010:2794-2805

Shipley B (2014) Measuring and interpreting trait-based selection versus meta-community effects during local community assembly. J Veg Sci 25:55-65

Sonnier G, Shipley B, Navas ML (2010) Quantifying relationships between traits and explicitly measured gradients of stress and disturbance in early successional plant communities. J Veg Sci 21:1014-1024

Sonnier G, Navas ML, Fayolle A, Shipley B (2012) Quantifying trait selection driving community assembly: a test in herbaceous plant communities under contrasted land use regimes. Oikos 121:1103-1111

Sterck FJ, Poorter L, Schieving F (2006) Leaf traits determine the growth-survival trade-off across rain forest tree species. Am Nat 167:758-765

Tilman D (1988) Plant strategies and the dynamics and structure of plant communities. Princeton University Press, Princeton

van Gelder HA, Poorter L, Sterck FJ (2006) Wood mechanics, allometry, and life-history variation in a tropical rain forest tree community. New Phytol 171:367-378

Violle C et al (2007) Let the concept of trait be functional! Oikos $116: 882-892$

Violle $C$ et al (2012) The return of the variance: intraspecific variability in community ecology. Trends Ecol Evol 27:244-252

Vitousek PM, Turner DR, Kitayama K (1995) Foliar nutrients during long-term soil development in Hawaiian montane rain forest. Ecology 76:712-720

Walters MB, Reich PB (1996) Are shade tolerance, survival, and growth linked-low light and nitrogen effects on hardwood seedlings. Ecology 77(3):841-853

Warton DI, Shipley B, Hastie T (2014) CATS regression-a modelbased approach to studying trait-based community assembly. Methods Ecol Evol 6:389-398

Wright IJ et al (2004) The worldwide leaf economics spectrum. Nature 428:821-827

Wright SJ et al (2010) Functional traits and the growth-mortality trade-off in tropical trees. Ecology 91:3664-3674 